\title{
Especies de Ciatostomas Resistentes al Albendazol en Equinos, Cuba
}

\author{
Species of Cyathostomin Resistant to Albendazole in Equines, Cuba \\ Josmel Salas-Romero ${ }^{1,4, *}$, Karel Gómez ${ }^{1,5, *}$, Yanise Chicoy², José C. Yero", \\ Edel Valle ${ }^{3}$, Anay Delgado-Martínez ${ }^{2}$, Jorge E. Salas-Romero ${ }^{1}$, Amilcar Arenal ${ }^{2}$
}

\section{Resumen}

Con el objetivo de evaluar la eficacia antihelmíntica de las presentaciones empleadas en los equinos de un rancho cubano, se realizó un test de reducción del recuento fecal de huevos de estróngilos (TRCFH) frente al albendazol y a la ivermectina, administrados vía oral. Se seleccionaron 33 equinos (albendazol 18; ivermectina 15). La reducción del conteo de huevos frente al albendazol varió entre $38 \%$ para el albendazol micronizado y $33 \%$ para el albendazol sulfóxido. La eficacia de la ivermectina fue del 100\%. Posteriormente, a cuatro equinos con altos recuentos de huevos de los grupos tratados con albendazol se les hizo una segunda desparasitación con ivermectina en el día 17 posterior a la dosificación inicial para colectar en las heces los nematodos resistentes al benzimidazol. Estos nematodos fueron identificados morfológicamente. Del total de nematodos colectados, se identificaron 493 pertenecientes a las especies Cylicocyclus nassatus, Cyathostomum catinatum, Cylicostephanus longibursatus, Cylicostephanus goldi y Cylicostephanus minutus, las cuales representaron el 78.5, 7.7, 6.5, 3.9 y 3.4\%, respectivamente. Este fue el primer reporte de $C$. minutus en equinos de Cuba. Se concluye que al menos cinco de las especies de ciatostomas fueron resistentes al albendazol.

Palabras clave: albendazol; ciatostomas; eficacia; resistencia

\section{Abstract}

This study aimed to evaluate the efficacy of anthelmintic used in an equine ranch in Cuba. The faecal egg count reduction test of Strongyles (FECRT) was used to evaluate albendazole and ivermectin. Thirty-three horses were selected (18 treated with albendazole,

\footnotetext{
${ }^{1}$ Laboratorio de Parasitología, ${ }^{2}$ Laboratorio de Bioquímica, Facultad de Ciencias Agropecuarias, Universidad de Camagüey Ignacio Agramonte Loynaz, Camagüey, Cuba

${ }^{3}$ Empresa Nacional para la Protección de la Flora y la Fauna, La Habana, Cuba

${ }^{4}$ E-mail: josmel.salas@reduc.edu.cu

${ }^{5}$ E-mail: karel.gomez@reduc.edu.cu

* Josmel Salas y Karel Gómez aportaron por igual en la realización del estudio
} 
15 treated with ivermectin). The egg count reduction for albendazole was $38 \%$ for micronized albendazole and $33 \%$ for albendazole sulfoxide. Ivermectin effectiveness was $100 \%$. Subsequently, four horses that showed high counts of eggs after the albendazole treatment were treated with ivermectin on day 17 after the initial deworming to collect resistant nematodes in the faeces. These nematodes were morphologically identified. It was possible to identified 493 specimens: Cylicocyclus nassatus, Cyathostomum catinatum, Cylicostephanus longibursatus, Cylicostephanus goldi and Cylicostephanus minutus which represented 78.5, 7.7, 6.5,3.9 and 3.4\% respectively. This has been the first report for Cylicostephanus minutus in equines in Cuba. It is concluded that at least five of species of cyathostomes were resistant to albendazole.

Key words: albendazole; cyathostomes; efficacy; resistance

\section{INTRODUCCIÓN}

Los programas tradicionales de control de parásitos incluyen el tratamiento periódico con antihelmínticos. Esta práctica y el empleo inadecuado de drogas antiparasitarias condujo a la aparición de nematodos resistentes en ganaderías equinas en todo el mundo (Kaplan y Nielsen, 2010; Scott et al., 2015).

La gran mayoría de los helmintos gastrointestinales de los equinos están representados por los miembros de la familia Strongylidae, con al menos 63 especies (Lichtenfels et al., 2008), de las cuales se reportan 24 en Cuba (Pérez-Vigueras, 1936; Varus y Valle, 1966). Por otra parte, solo se dispone en el país de tres publicaciones referidas a la eficacia antihelmíntica de la ivermectina en el equino (Arece et al., 2002; Salas-Romero et al., 2014, 2017), mientras que un estudio reciente se refiere a la baja eficacia del albendazol (ABZ) en el control de los ciatostomas de los equinos de la ciudad de Camagüey y zonas aledañas (SalasRomero et al., 2017), sin indicar las especies resistentes.

La resistencia a los benzimidazoles (BZ) es la más frecuente en diversos países. El 95\% de las poblaciones de ciatostomas de los rebaños equinos en el sudeste de EEUU son resistentes a los BZ (Kaplan, 2004). Se conoce que una mutación del gen de la beta tubulina en nematodos de rumiantes y en varias especies comunes de ciatostomas les confiere la capacidad de resistencia a este grupo farmacológico (Garcia et al., 2013; Matthews, 2014). En Cuba, la falta de presentaciones comerciales de antiparasitarios de uso en equinos, ha obligado a veterinarios y propietarios a emplear las presentaciones indicadas en otras especies de hospederos para el control de las helmintiasis (Salas-Romero et al., 2017).

Los helmintos intestinales de los caballos son mayormente estudiados por medio de la necropsia helmintológica (Reinemeyer et al., 2015), lo que resulta considerablemente costoso. Por otro lado, el examen de la nematofauna posterior a la desparasitación ha sido realizada de forma exitosa por investigadores de Ucrania (Kuzmina et al., 2016). Por estas razones, el objetivo de este estudio fue evaluar la eficacia de los antihelmínticos empleados en el control de los estróngilos de los caballos del rancho Turiguanó, en Cuba, y determinar las especies resistentes al albendazol.

\section{Materiales y Métodos}

\section{Ubicación y Animales}

El trabajo se llevó a cabo entre noviembre de 2015 y enero de 2016 en el rancho - 
Turiguanó, el cual está situado a $1 \mathrm{~km}$ de la costa norte del municipio Morón, actual provincia de Ciego de Ávila, Cuba. El rancho cuenta con caballos de la raza Quarter Horse.

Los registros veterinarios del centro indicaban que en los últimos tres años se realizaron entre tres y cuatro desparasitaciones anuales a toda la masa equina. Además, el personal veterinario a través de entrevistas refirió que dicha práctica se realiza desde hace más de 10 años. En este periodo, salvo una vez en la cual se empleó Baymec (Bayer, Alemania), los equinos se desparasitaron con Labiomec (ivermectina), Albendazol (albendazol micronisado) o Labiozol (albendazol sulfóxido), producidos por Labiofam, Cuba. La posible alternancia o selección de los medicamentos siempre estuvo determinada por su disponibilidad en el mercado nacional.

\section{Muestras de Heces}

Todos los animales fueron desparasitados 86 días antes del muestreo inicial, como parte del programa de control antihelmíntico rutinario. Se recolectaron muestras de heces a 65 equinos, de los cuales se seleccionaron 33 yeguas vacías o con al menos cuatro meses de gestación (debido al efecto teratogénico del albendazol al inicio de la gestación), con edades entre 3 y 14 años, que presentaron 675 huevos de estróngilos por gramo de heces (hpg) como mínimo.

Se recolectó una segunda muestra de heces $(20 \mathrm{~g})$ del recto de las yeguas seleccionadas en el primer día del estudio (día 0 , día en que se administró el antihelmíntico) y una tercera muestra 14 días después. Las muestras se transportaron en una nevera refrigerada hasta el Laboratorio de Parasitología de la Universidad de Camagüey "Ignacio Agramonte Loynaz" y procesadas antes de las $72 \mathrm{~h}$ posteriores a su toma. El conteo fecal de huevos se realizó por la técnica de McMaster con una sensibilidad de $25 \mathrm{hpg}$ (Nielsen et al., 2013).

\section{Tratamientos}

Se evaluó la eficacia de los dos principios activos de uso frecuente en los equinos de Rancho Turiguanó: albendazol en sus dos presentaciones comerciales, Albendazol (ABZ-M) (albendazol micronizado) y Labiozol (ABZ-S) (albendazol sulfóxido), ambos en dosis de $10 \mathrm{mg} / \mathrm{kg}$ de peso vivo (PV) y la ivermectina (IVM) al $1 \%$ (Labiomec ${ }^{\circledR}$ ) a razón de $0.2 \mathrm{mg} / \mathrm{kg}$ PV.

Los antiparasitarios se administraron vía oral, teniéndose 6 yeguas para el ABZ-M, 12 yeguas para el ABZ-S y 15 yeguas para IVM. Los grupos de tratamientos fueron conformados a elección del propietario.

\section{Análisis de los Datos}

La eficacia del tratamiento se determinó mediante el test de reducción de conteo fecal de huevos de estróngilos (TRCFH), basado en la diferencia entre las medias aritméticas del número de hpg antes y después del tratamiento, expresada en porcentaje.

Los resultados del TRCFH se clasificaron de acuerdo con lo recomendados para IVM (Nielsen et al., 2013) y en el caso del $\mathrm{ABZ}$ se emplearon los valores sugeridos para los BZ fenbendazol y oxibendazol (Nielsen et al., 2013), donde:

- IVM: (1) Presencia de resistencia, cuando la reducción del conteo fecal de huevos (RCFH) es $<95 \%$; (2) Sospechoso de resistencia, cuando la RCFH es entre 95 y 98\%; (3) Susceptible (ninguna evidencia de resistencia), cuando la RCFH es $>98$ y $99 \%$; (4) Eficacia esperada, cuando la RCFH es $>99.0 \%$.

- ABZ: (1) Presencia de resistencia, cuando la RCFH es $<90 \%$; (2) Sospechoso de resistencia, cuando la RCFH es entre 90 y 95\%; (3) Susceptible (ninguna evidencia de resistencia) cuando la RCFH es entre $>95$ y $98 \%$; (4) Eficacia esperada, cuando la RCFH es $>98 \%$. 
Cuadro 1. Test de reducción del conteo fecal de huevos (hpg) de estróngilos en equinos dosificados con albenzadol (ABZ) e ivermectina (IVM) en yeguas del rancho Turiguanó, Ciego de Ávila (Cuba)

\begin{tabular}{lccccc}
\hline Medicamento & Yeguas & $\begin{array}{c}\text { HPG Día 0 } \\
\text { Media (rango) }\end{array}$ & $\begin{array}{c}\text { HPG Día 14 } \\
\text { Media (rango) }\end{array}$ & $\begin{array}{c}\mathrm{RCFH}^{2} \\
(\%)\end{array}$ & $\begin{array}{c}\text { LIC }^{3} \\
(\%)\end{array}$ \\
\hline ABZ-M & 6 & $\begin{array}{c}2413 \\
(1025-3900)\end{array}$ & $\begin{array}{c}1496 \\
(400-2675)\end{array}$ & 38 & 8 \\
ABZ-S & 12 & $\begin{array}{c}2744 \\
(675-4200)\end{array}$ & $\begin{array}{c}1811 \\
(325-3650)\end{array}$ & 33 & 4 \\
\hline Total ABZ & 18 & 2633 & 1718 & 35 & 7 \\
\hline IVM & 15 & $\begin{array}{c}2575 \\
(1125-3775)\end{array}$ & $\begin{array}{c}3 \\
(0-25)\end{array}$ & 99.87 & 99 \\
\hline
\end{tabular}

${ }^{1}$ ABZ-M: albendazol micronizado; ABZ-S: albendazol sulfóxido

2 Porcentaje de reducción del conteo fecal de huevos (\% RCFH)

${ }^{3}$ Límite inferior del intervalo de confianza 95\%

\section{Identificación de Helmintos Resistentes}

Se seleccionaron cuatro equinos cuya reducción del hpg mostró resistencia antihelmíntica al ABZ (con valores entre 1075 y $1850 \mathrm{hpg})$. Estos animales se desparasitaron con IVM el día 17 (tres jornadas después de confirmado el hpg al día 14) con el objetivo de colectar la nematofauna que sobrevivió al tratamiento con ABZ (Kuzmina et al., 2016).

La colecta de los nematodos expulsados se realizó en dos tomas entre las 24 y a las $48 \mathrm{~h}$ del tratamiento con IVM, siguiendo la variación en la dinámica de expulsión (Kuzmina et al., 2005). Los helmintos se lavaron con $\mathrm{NaCl} 0.9 \%$ durante $2 \mathrm{~h}$ y posteriormente se almacenaron en alcohol al 70\%.

Para la clasificación de los helmintos, los parásitos se sumergieron en lactofenol por 30 min y luego se colocaron entre porta y cubre objeto para ser observados en un microscopio Leica DM 300. La identificación de los especímenes se basó en las claves de clasificación de Lichtenfels et al. (2008) y
Tolliver (2001). Las fotos fueron tomadas con una cámara digital Sony DSC-W800.

El material comprobatorio está depositado en las Colecciones Zoológicas del Instituto de Ecología y Sistemática del Ministerio de Ciencia, Tecnología y Medio Ambiente de Cuba, La Habana (Números de depósito: $11.7044 ; 11.7045 ; 11.7046 ; 11.7048$ y 11.7049).

\section{Resultados}

El muestreo pretratamiento solo mostró dos equinos con valores inferiores a $500 \mathrm{hpg}$, pero ninguno con valor cero. La media de hpg fue de 2479, con un rango de 400 a 4200. En esta población, el $40 \%$ de los equinos expulsó al medio el $80 \%$ de los huevos de estróngilos contabilizados en el material fecal.

En el proceso de entrevista, el personal veterinario del rancho refirió que habían notado que los equinos expulsaban un mayor 


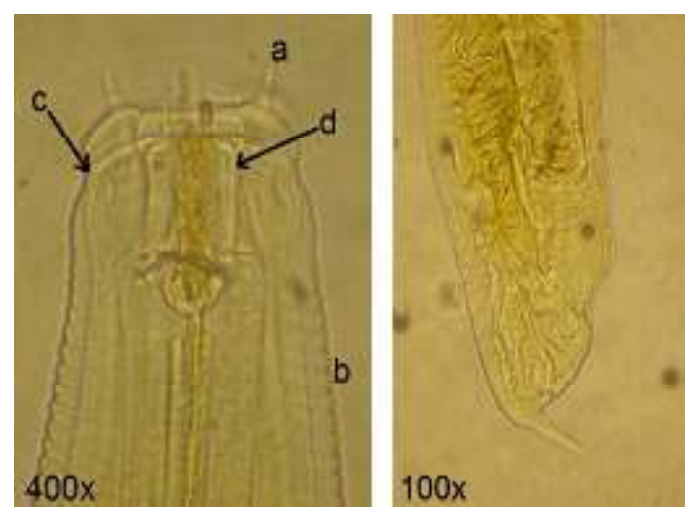

Figura 1. Especímen de Cylicostephanus minutus (Izquierda: cabeza; Derecha: cola de la hembra)

número de parásitos en el material fecal asl desparasitar con ivermectina en comparación con los tratados con albendazol, y que al tratar estos equinos con ivermectina pasadas una o dos semanas, volvían a expulsar nematodos en el material fecal.

El ABZ se evaluó en 18 caballos y mostró una eficacia del 35\% (Cuadro 1), variando entre el 33 y el 38\% de eficacia para sus dos presentaciones. En el caso de la IVM se observó una eficacia de1 99.87\% (Cuadro 1), donde solo se detectaron dos yeguas con valores postratamiento de $25 \mathrm{hpg}$.

Los estróngilos identificados pertenecen a los géneros Cylicocyclus, Cyathostomum y Cylicostephanus. De un total de 1631 nematodos colectados se llegó a identificar 493, pertenecientes a las especies Cylicocyclus nassatus (Looss, 1900), Cyathostomum catinatum (Looss, 1900), Cylicostephanus longibursatus (Yorke y Macfie, 1918), Cylicostephanus goldi (Boulenger, 1917) y Cylicostephanus minutus (Yorke y Macfie, 1918), las cuales representaron el 78.5, 7.7, 6.5, 3.9 y $3.4 \%$, respectivamente. Es importante resaltar que Cylicostephanus minutus (Figura 1) no había sido reportado con anterioridad en caballos en Cuba (Los nematodos no identificados se conservaron en alcohol al $70 \%$ a $-20{ }^{\circ} \mathrm{C}$ para estudios posteriores de secuenciamiento del gen de la $\beta$ tubulina).

En Cylicostephanus minutus, las papilas submediales se encuentran en el medio entre el cuello de las fauces del extremo anterior de la boca y se asemejan a las antenas de un caracol (Fig. 1-a), la cutícula muestra estriaciones (Fig. 1-b) y presenta una ligera contracción en el cuello (Fig. 1-c). Las paredes de la cápsula bucal se observan simétricas y rectas hasta la salida en las fauces del extremo anterior de la boca (Fig. 1d). Es uno de los ciatostomas más pequeños. La cola de las hembras es gruesa, en cuyo extremo presenta una pequeña púa o espiga ligeramente inclinada (Fig. 1). Los machos presentan una pequeña bolsa copulatoria que es aproximadamente tan larga como ancha (no se muestras imágenes por la baja calidad de la cola de los machos observados).

\section{Discusión}

El promedio de hpg encontrado en el presente estudio constituye el mayor valor reportado en equinos en Cuba, con valores de media previos que oscilaron entre 1445 (Salas-Romero et al., 2014) y 1630 (SalasRomero, 2012). Por otra parte, la distribución de la liberación de huevos de estróngilos en el material fecal constituye uno de los elementos en los cuales es basada la desparasitación selectiva en estos hospederos (Nielsen et al., 2014; Nielsen, 2015).

En países de climas templados, el $80 \%$ de los huevos de estróngilos son expulsados por el $11-20 \%$ de los caballos del rebaño (Kaplan y Nielsen, 2010; Wood et al., 2012; Lester et al., 2013; Relf et al., 2013), por lo que al desparasitar una pequeña parte del hato se logra disminuir teóricamente la carga contaminante en las pasturas manteniendo una gran población parasitaria en refugio (Nielsen et al., 2014; Nielsen, 2015). Sin embargo, en el presente estudio para estas condiciones, el $80 \%$ de la ovoposición total se presentó en 
cerca de la mitad de los equinos, y la otra parte presentaron conteos de hasta $1223 \mathrm{hpg}$, valor que supera las medias reportadas para la mayoría de las poblaciones equinas en el mundo (Kaplan y Nielsen, 2010; Wood et al., 2012; Lester et al., 2013; Relf et al., 2013).

Actualmente, los valores sugeridos por la literatura como punto de corte en la desparasitación selectiva en equinos se encuentran entre 200 y $500 \mathrm{hpg}$ (Nielsen et al., 2014; Nielsen, 2015). Solo dos animales presentaron valores inferiores en el momento del diagnóstico inicial, lo que muestra la necesidad de determinar los posibles puntos de corte del hpg para la desparasitación selectiva en equinos de países tropicales.

La resistencia antihelmíntica de los ciatostomas a los BZs se informó por primera vez en la década de 1960, como consecuencia de las estrategias de control contra los grandes estróngilos (Peregrine et al., 2014). Actualmente la resistencia a los BZs resulta la más prevalente en los ciatostomas (Traversa et al., 2012), reportándose en al menos 14 países (Peregrine et al., 2014). Por su parte, la periodicidad de los tratamientos antihelmínticos en el Rancho Turiguanó imprime una alta presión de selección sobre la población parasitaria (Scott et al., 2015).

Otras investigaciones reportan $100 \%$ de efectividad del ABZ en equinos en dosis de 5 y $10 \mathrm{mg} / \mathrm{kg}$ de peso vivo en Ucrania y Sudán, respectivamente (Kuzmina y Kharchenko, 2008; Imam et al., 2010). Esta situación es similar a los resultados del TRCFH en San Vicente y Triángulo Tres, reportados en Camagüey (Salas-Romero et al., 2017) con eficacia de 99.9 y $99.7 \%$, respectivamente, utilizando la presentación local de ABZ-M, lo que demuestra que esta presentación puede alcanzar una alta eficacia ante poblaciones de helmintos susceptibles. No obstante, la falta de presentaciones formuladas específicamente para caballos en Cuba, puede haber sido un elemento catalizador de resistencia antihelmíntica en esta especie, similar a lo sugerido por Stratford et al. (2014), quienes refieren que el empleo de antihelmínticos parenterales para bovinos pudo influir en el desarrollo de resistencia a las lactonas macrocíclicas (LM) en el Reino Unido. Por otro lado, la alta eficacia de la IVM en el control de los nematodos de los equinos se informó con anterioridad en Cuba (Arece et al., 2002; Salas-Romero et al., 2014) y en el mundo (Fangama et al., 2013; Pérez-Álvarez et al., 2013).

La situación de la resistencia antihelmíntica en los caballos de Cuba resulta más compleja que en otras partes del mundo, ya que los criadores, cuidadores y veterinarios de equinos en el país, solo tienen acceso a dos principios activos de antihelmínticos, los cuales, por demás, no constituyen presentaciones fabricadas para su administración a esta especie. Además, el traslado a ferias y eventos hípicos o de rodeo dentro del país o su empleo como medio de transporte, hace que los equinos presenten un potencial diseminador de la resistencia, muy superior a otras especies animales.

Los ciatostomas están representados por cerca de 51 especies (Matthews, 2014), donde 12 se identifican como las más prevalentes, constituyendo el $99 \%$ del total de la población de ciatostomas en el intestino de los equinos (Kaplan, 2002; Kuzmina y Kharchenko, 2008).

En Ukrania, las especies Cylicocyclus nassatus, Cyathostomum catinatum y Cylicostephanus longibursatus resultaron ser las especies resistentes al albendazol más abundantes $(5 \mathrm{mg} / \mathrm{kg})$, representando el 68.6, 18.1 y $5.8 \%$ de los colectados pos-tratamiento con una LM, respectivamente (Kuzmina y Kharchenko, 2008), aunque también se detectaron ejemplares resistentes de $C$. gold $i$ y $C$. minutus. Estas especies se encuentran entre las más frecuentes en la necropsia en equinos tratados previamente con fenotiazina, pirantel, tiabendazol y febendazol y diagnosticados con resistencia a estos productos en equinos de Kentucky, EEUU (Lyons et al., 2007). 
En Cuba, solo se dispone de dos estudios que identificaron la nematofauna equina local, el primero en 1936 (Pérez-Vigueras, 1936) y el segundo en 1966 (Varus y Valle, 1966). Entre ambos se identificaron 24 especies de estróngilos.

El hallazgo de Cylicostephanus minutus por vez primera, unido a la detección en 2012 de Poteriostomum sp (Salas-Romero et al., 2014), confirma lo planteado por dichos autores sobre la probable presencia de un mayor número de especies presentes en los equinos de Cuba, aún por identificar.

Este trabajo constituye el segundo informe de baja eficiencia antihelmíntica en equinos en Cuba, luego de lo reportado en Camagüey por Salas-Romero et al. (2017). Los riesgos que implica el uso extendido de presentaciones parenterales de antihelmínticos, desarrollados para su aplicación en otras especies y otras vías de administración, y la falta de presentaciones comerciales para equinos en Cuba, unido a los hallazgos del presente estudio, permiten suponer que la resistencia antihelmíntica podría ser un fenómeno ampliamente difundido en los rebaños equinos del país.

\section{Agradecimientos}

Es preciso agradecer la colaboración brindada por los investigadores ucranianos Tetiana A. Kuzmina y Vitaliy Kharchenko en la identificación de algunos especímenes.

\section{Literatura Citada}

1. Arece J, Rojas F, González E, Cáceres O. 2002. Eficacia de Labiomec ${ }^{\circledR}$ en el parasitismo en ovinos, terneros y equinos en condiciones de producción. Pastos y Forrajes 25: 223-229.

2. Fangama M, Seri H, Suliman S, Imam S, Mozamel E. 2013. Comparative efficacy evaluation of moxidectin and ivermectin injectable formulation against helminthes infestation of donkeys (Equus asinus) in Sudan. Assiut Vet Med J 59: 1-8.

3. Garcia A, Brady HA, Nichols WT, Prien S. 2013. Equine cyathostomin resistance to fenbendazole in Texas horse facilities. J Equine Vet Sci 33: 223-228. doi: 10.1016/j.jevs.2012.06.005

4. Imam SM, Seri HI, Hassan T, Tigani TA, Zolain HB, Abakar AD. 2010. Therapeutic efficacy evaluation of anthelmintics activity of albendazole and ivermectin drench formulations in donkeys in Darfur, Sudan. Vet Arhiv 80: 585-595.

5. Kaplan RM, Nielsen MK. 2010. An evidence-based approach to equine parasite control: It ain't the 60s anymore. Equine Vet Educ 22: 306-316. doi: 10.1111/j.2042-3292.2010.00084.x

6. Kaplan RM. 2002. Anthelmintic resistance in nematodes of horses. Vet Res 33: 491-507. doi: 10.1051/ vetres:2002035

7. Kaplan RM. 2004. Drug resistance in nematodes of veterinary importance: a status report. Trends Parasitol 20: 477481. doi: http://dx.doi.org/10.1016/ j.pt.2004.08.001

8. Kuzmina TA, Dzeverin I, Kharchenko $V A .2016$. Strongylids in domestic horses: influence of horse age, breed and deworming programs on the strongyle parasite community. Vet Parasitol 227: 56-63. doi: 10.1016/j.vetpar.2016.07.024

9. Kuzmina TA. Kharchenko VA. Starovir AI. Dvojnos GM. 2005. Analysis of the strongylid nematodes (Nematoda: Strongylidae) community after deworming of brood horses in Ukraine. Vet Parasitol 131: 283-290. doi: 10.1016/j.vetpar.2005.05.010

10. Kuzmina TA. Kharchenko VO. 2008. Anthelmintic resistance in cyathostomins of brood horses in Ukraine and influence of anthelmintic treatments on strongylid community structure. Vet Parasitol 154: 277-288. doi: 10.1016/j.vetpar. 2008.03.024 
11. Lester HE, Spanton J, Stratford CH, Bartley DJ, Morgan ER, Hodgkinson JE, Coumbe K, et al. 2013. Anthelmintic efficacy against cyathostomins in horses in Southern England. Vet Parasitol 197: 189-196. doi: 10.1016/j.vetpar.2013.06.009

12. Lichtenfels JR, Kharchenko VA, Dvojnos GM. 2008. Illustrated identification keys to strongylid parasites (Strongylidae: Nematoda) of horses, zebras and asses (Equidae). Vet Parasitol 156: 4-161. doi: 10.1016/ j.vetpar.2008.04.026

13. Lyons ET, Tolliver SC, Collins SS. 2007. Study (1991 to 2001) of drugresistant Population B small strongyles in critical tests in horses in Kentucky at the termination of a 40-year investigation. Parasitol Res 101: 689-701. doi: 10.1007/ s00436-007-0535-6

14. Matthews JB. 2014. Anthelmintic resistance in equine nematodes. Int $\mathrm{J}$ Parasitol Drugs Resist 4: 310-315. doi: 10.1016/j.ijpddr.2014.10.003

15. Nielsen M, Pfister K, von SamsonHimmelstjerna G. 2014. Selective therapy in equine parasite control Application and limitations. Vet Parasitol 202: 95-103. doi: 10.1016/j.vetpar. 2014.03.020

16. Nielsen MK, Mittel L, Grice A, Erskine M, Graves E, Vaala W, Tully $R C$, et al. 2013. AAEP parasite control guidelines. AAEP Parasite Control Subcommittee of the AAEP Infectious Disease Committee. 24 p. [Internet]. Disponible en: http://www.aaep.org/ custdocs/Parasite Control Guidelines Final.pdf

17. Nielsen MK. 2015. Universal challenges for parasite control: a perspective from equine parasitology. Trends Parasitol 31: 282-284. doi: 10.1016/ j.pt.2015.04.013

18. Peregrine AS, Molento MB, Kaplan RM, Nielsen MK. 2014. Anthelmintic resistance in important parasites of horses: does it really matter? Vet
Parasitol 201: 1-8. doi: 10.1016/ j.vetpar.2014.01.004

19. Pérez-Álvarez S, Rojas-Mujica K, Bello H, Villoria D, Morales A. 2013. Comparative study of two therapies pharmacological based a ivermectin and febendazol by strongyles control intestinal in thorougbreds horses. J Vet Sci Technol 4(2). doi: 10.4172/21577579.1000144

20. Pérez-Vigueras I. 1936. Notas sobre la fauna parasitológica de Cuba. En: Memorias de la Sociedad Cubana de Historia Natural 10: 53-86.

21. Reinemeyer CR, Prado JC, Nielsen MK. 2015. Comparison of the larvicidal efficacies of moxidectin or a five-day regimen of fenbendazole in horses harboring cyathostomin populations resistant to the adulticidal dosage of fenbendazole. Vet Parasitol 214: 100107. doi: 10.1016/j.vetpar.2015.10.003

22. Relf V, Morgan E, Hodgkinson J, Matthews J. 2013. Helminth egg excretion with regard to age, gender and management practices on UK Thoroughbred studs. Parasitology 140: 641-652. doi: 10.1017/S0031182012001941

23. Salas-Romero J, Gómez-Cabrera K, Molento M, Lyon ET, DelgadoMartinez A, Gonzalez L, Arenal A, Nielsen M. 2017. Efûcacy of two extra-label anthelmintic formulations against equine strongyles in Cuba. Vet Parasitol Reg Stud Rep 8: 39-42. doi: 10.1016/j.vprsr.2017.01.010

24. Salas-Romero J, Mencho JD, Guerra Y, Mencho JC. 2014. Prevalencia de nematodos intestinales y eficacia de

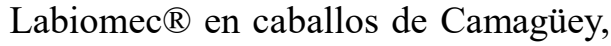
Cuba. Rev Salud Anim 36: 152-158.

25. Salas-Romero J. 2012. Evaluación del comportamiento de los nematodos gastrointestinales de los equinos en Camagüey. Tesis de Maestría. Cuba: Univ de Camagüey «Ignacio Agramonte y Loynaz». $94 \mathrm{p}$. 
26. Scott I, Bishop R, Pomroy W. 2015. Anthelmintic resistance in equine helminth parasites-a growing issue for horse owners and veterinarians in New Zealand? N Z Vet J 63: 188-198. doi: 10.1080/00480169.2014.987840

27. Stratford C, Lester H, Pickles K, McGorum B, Matthews J. 2014. An investigation of anthelmintic efficacy against strongyles on equine yards in Scotland. Equine Vet J 46: 17-24. doi: 10.1111/evj.12079

28. Tolliver CS. 2001. A practical method of identification of the North American cyathostomes (small strongyles) in equids in Kentucky. Kentucky Agricultural Experiment Station, University of Kentucky. [Internet]. Disponible en: http://www2.ca.uky.edu/ agcomm/pubs/sr/sr2000-1/intro.pdf
29. Traversa D, Castagna G, von SamsonHimmelstjerna G, Meloni S, Bartolini R, Geurden T, Pearce, et al. 2012. Efficacy of major anthelmintics against horse cyathostomins in France. Vet Parasitol 188: 294-300. doi: 10.1016/ j.vetpar.2012.03.048

30. Varus V, Valle MT. 1966. Nuevos registros de nematodos que parasitan en caballos (Equus caballus Linné) de Cuba. Poeyana Serie A 27: 1-10.

31. Wood E, Matthews J, Stephenson S, Slote M, Nussey D. 2012. Variation in fecal egg counts in horses managed for conservation purposes: individual egg shedding consistency, age effects and seasonal variation. Parasitology 140: 115128. doi: 10.1017/S003118201200128X 